\title{
Hydration of $\operatorname{Kr}(\mathrm{aq})$ in Dilute and Concentrated Solutions
}

\author{
Mangesh I. Chaudhar* \\ Center for Biological and Material Sciences, Sandia National Laboratories, Albuquerque, NM, 87123 \\ Dubravko Sabd \\ Department of Chemistry, New York University, New York, NY 10003 \\ Lawrence R. Pratt团 \\ Department of Chemical and Biomolecular Engineering, Tulane University, New Orleans, LA, 70118 \\ Susan B. Remp£ $\S^{\S}$ \\ Center for Biological and Material Sciences, Sandia National Laboratories, Albuquerque, NM, 87185
}

(Dated: October 3, 2018)

\begin{abstract}
Molecular dynamics simulations of water with both multi-Kr and single $\mathrm{Kr}$ atomic solutes are carried out to implement quasi-chemical theory evaluation of the hydration free energy of $\operatorname{Kr}(\mathrm{aq})$. This approach obtains free energy differences reflecting $\mathrm{Kr}-\mathrm{Kr}$ interactions at higher concentrations. Those differences are negative changes in hydration free energies with increasing concentrations at constant pressure. The changes are due to a slight reduction of packing contributions in the higher concentration case. The observed $\mathrm{Kr}-\mathrm{Kr}$ distributions, analyzed with the extrapolation procedure of Krüger, et al., yield a modestly attractive osmotic second virial coefficient, $B_{2} \approx-60 \mathrm{~cm}^{3} / \mathrm{mol}$. The thermodynamic analysis interconnecting these two approaches shows that they are closely consistent with each other, providing support for both approaches.
\end{abstract}

Keywords: hydrophobic interactions, quasi-chemical theory, osmotic second virial coefficient

\section{INTRODUCTION}

Here we analyze the hydration of the classic hydrophobic solute krypton (Kr) in liquid water solution, including at elevated concentrations that permit $\mathrm{Kr}-\mathrm{Kr}$ encounters to be assessed. $\mathrm{Kr}(\mathrm{aq})$ is specifically interesting for several reasons. Firstly, $\mathrm{Kr}$ is isoelectronic with $\mathrm{Rb}^{+}$, which has been analyzed in detail. 1 Secondly, $\operatorname{Kr}(\mathrm{aq})$ has been a target of sophisticated experimental structural work.$^{2 \mid 3}$ Finally, $\operatorname{Kr}(\mathrm{aq})$ has been the focus of intense theoretical attention, 4 but the statistical mechanical theory has become much more sophisticated in recent years. 8

Here, we use molecular dynamics simulations to study $\operatorname{Kr}(\mathrm{aq})$ solutes utilizing quasi-chemical theory 911 This theory rigorously decomposes the free energy of hydration into packing, outer-shell and chemical components, is independent of van der Waals assumptions, $\frac{8}{,}$ and thus physically clarifies the hydrophobic solubility of $\mathrm{Kr}$ in water. Recent theoretical work has returned to the basic problem of evaluating the osmotic second virial coefficients, $B_{2}$, for simple hydrophobic solutes in water. 1213 We use the new statistical thermodynamic results here to reanalyze that important problem.

The next section specifies the technical data for the simulations carried out here. Succeeding sections lay out the quasi-chemical theory employed, then present results and discussion.

\section{COMPUTATIONAL METHODS}

The structural properties of interest are computed using the GROMACS molecular dynamics simulation package.14 Simulations were carried out in the isothermal-isobaric $(N p T)$ ensemble at $T=300 \mathrm{~K}$ and $p=1 \mathrm{~atm}$. The simulation cell was a cube, and standard periodic boundary conditions were used to mimic bulk solution.

A single $\mathrm{Kr}$ atom and $16 \mathrm{Kr}$ in water were simulated separately to study the effects of solute concentration. The mole fraction of $1 \mathrm{Kr}$ in 1000 water molecules is comparable to concentrations used in experimental hydration free energy studies,, 5 the higher concentration case is likely a supersaturated solution, but that did not lead to problematic behavior. After energy minimization and 1 ns of volume equilibration, the simulations were extended to 20 ns of production run. Bonds involving hydrogens were fixed by the LINCS algorithm. The equations of motions were integrated using a time step of $1 \mathrm{fs}$. Temperature and pressure were maintained using a Nose-Hoover ${ }^{16}$ thermostat and a Parinello-Rahman 17 barostat. Interactions between water molecules were described by the SPC/E model ${ }^{18}$ The Kr atoms were treated as Lennard-Jones (LJ) spheres with parameters $\sigma_{\mathrm{KrKr}}=3.935 \AA$ and $\epsilon_{\mathrm{KrKr}}=0.4342 \mathrm{kcal} / \mathrm{mol} !^{19}$ For $\mathrm{KrO}$ interactions, the mixing rules of the OPLS forcefield were followed ${ }^{14}$ Configurations were sampled every $0.5 \mathrm{ps}$. 


\section{THEORY}

The hydration free energy of $\operatorname{Kr}(\mathrm{aq})$ was computed using quasi-chemical theory (QCT), 20121

$$
\begin{aligned}
& \beta \mu_{\mathrm{Kr}}^{(\mathrm{ex})}\left(\rho_{\mathrm{Kr}}, p, T\right)=-\ln p^{(0)}\left(n_{\lambda}=0\right) \\
& +\ln \left\langle e^{\beta \varepsilon} \mid n_{\lambda}=0\right\rangle+\ln p\left(n_{\lambda}=0\right) .
\end{aligned}
$$

This equation involves packing, outer-shell and chemical contributions on the right-hand side. Those contributions are discussed further below. Eq. (1) has been used to study hydration free energies of water, ${ }^{22} \mathrm{CH}_{4}, 25$ $\mathrm{CF}_{4} / \mathrm{C}\left(\mathrm{CH}_{3}\right)_{4}, \frac{8}{,}$, hydrogen ${ }^{26]}$ and carbon dioxide. ${ }^{27} \mathrm{We}$ evaluate the free energy contributions numerically by analyzing simulation trajectories.

Recognizing $p^{(0)}\left(n_{\lambda}=0\right)$ as the probability of finding an empty inner shell at an arbitrary point in the fluid, the term $-\ln p^{(0)}\left(n_{\lambda}=0\right)$ assesses the free energy required to form the corresponding cavity. This packing contribution was estimated by random placement of 30,000 spheres of radius $\lambda$, scoring the fraction of those placements conforming to the 'vacant' event.

The middle term represents interactions of outer-shell solvent molecules with a solute in a vacant inner shell. This outer-shell contribution is approximated as

$$
\begin{aligned}
\ln \left\langle e^{\beta \varepsilon} \mid n_{\lambda}=0\right\rangle \approx \beta\langle\varepsilon| n_{\lambda} & =0\rangle \\
& +\beta^{2}\left\langle\delta \varepsilon^{2} \mid n_{\lambda}=0\right\rangle / 2 .
\end{aligned}
$$

This is the important simplification of quasi-chemical theory that the construction of Eq. (1) is designed to achieve. With this ingredient, quasi-chemical theory is seen to be a generalization of the van der Waals picture of liquids. ${ }^{2128}$ This gaussian approximation has been welltested for the substantial list of applications noted,,$[22]+27$ and including electrolyte solutions. 29

The right-most term of Eq. (1) is the 'chemical' contribution to the hydration free energy. It can also be recognized as the free energy regained with relaxation of the constraint associated with the successful formation of a cavity at the initial step.21 This contribution is estimated directly by observing $\mathrm{Kr}$ atoms present in the simulation.

The individual contributions of Eq. (1) depend on $\lambda$, which sets the boundary between inner- and outer-shell solvent molecules. The excess chemical potential, $\mu_{\mathrm{Kr}}^{(\mathrm{ex})}$, should be independent of that boundary. Here, we evaluate $\mu_{\mathrm{Kr}}^{(\mathrm{ex})}$ for a range of $\lambda$ covering as much structural data as statistically feasible.

\section{RESULTS AND DISCUSSION}

Characterizing the solution structure around the $\mathrm{Kr}$ atoms, FIG. 1 shows several radial distribution functions. Both $\mathrm{KrO}$ and $\mathrm{KrH}$ distributions display a principal maximum at about $3.7 \AA$. The water structure, $g_{\mathrm{OO}}(r)$ (not

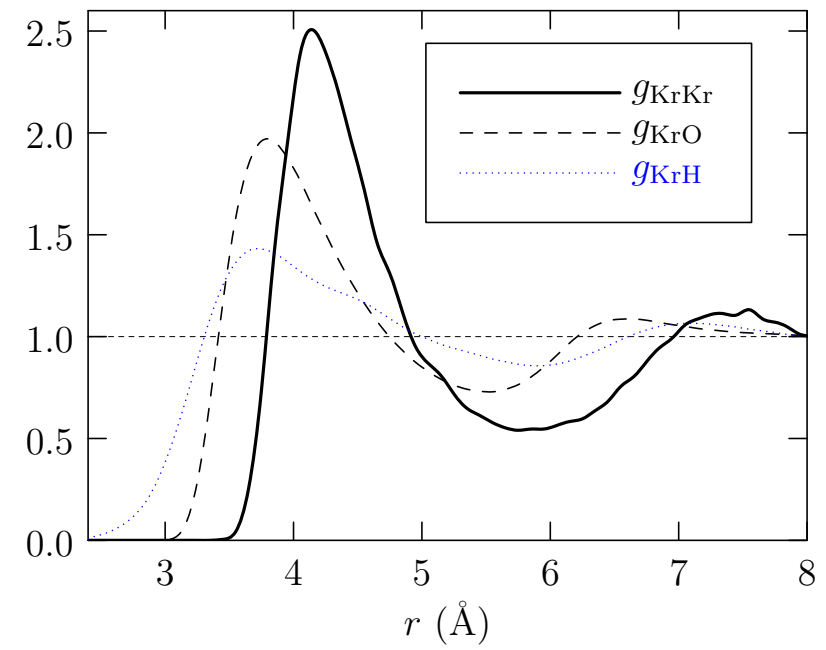

FIG. 1: Radial distribution functions observed for the aqueous Kr solution at elevated concentration, $n_{\mathrm{Kr}}=16$. The water structure, $g_{\mathrm{OO}}(r)$ (not shown), is closely similar to that of bulk water.

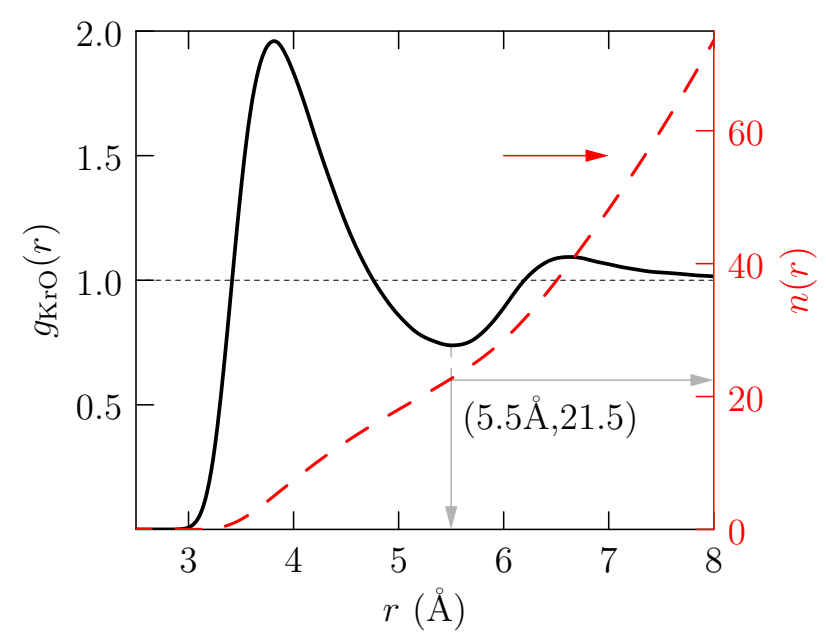

FIG. 2: Higher detail for $g_{\mathrm{KrO}}(r)$ around $\mathrm{Kr}$ atoms (for the $n_{\mathrm{Kr}}=16$ case). The red dashed line is the running coordination number $n(r)$, indicating an average O-occupancy of 21.5 for the sphere within the first minimum defined by a first inner-shell radius of $\lambda=5.5 \AA$. The principal minimum is not unusually deep, and outside the principal maximum, the fluid is only weakly structured.

shown), is largely unaffected by the $\mathrm{Kr}$ for these calculations. A more exclusive view of the hydration structure (FIG. 2) shows that the average O-occupancy of the sphere within the first minimum for first inner-shell boundary of $5.5 \AA$ is 21.5 . The principal minimum is not unusually deep, and outside the principal maximum the fluid is only weakly structured. Neighborship decomposition (FIG. 3) shows that the principal maximum of $g_{\mathrm{KrO}}(r)$ is saturated by fewer than 10 nearest neighbors.

For solutes of van der Waals type generically, the nat- 


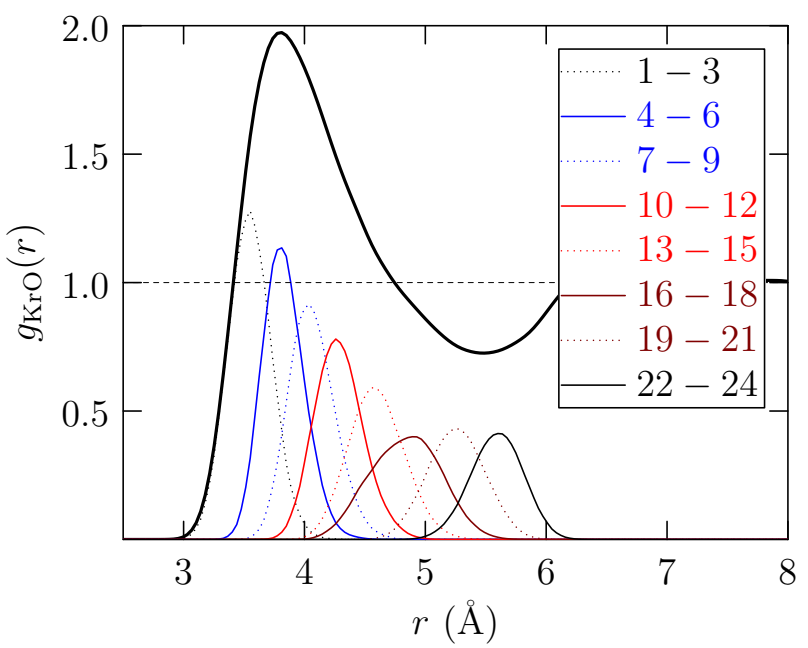

FIG. 3: Neighborship decomposition of the radial distribution $g_{\mathrm{KrO}}(r)$, from the $n_{\mathrm{Kr}}=1$ case. The maximum is located at $r \approx 3.8 \AA$. Note that the principal maximum of $g_{\mathrm{KrO}}(r)$ is saturated by fewer than 10 nearest neighbors. See also FIG. 2. Outside the principal maximum, the fluid is only weakly structured.

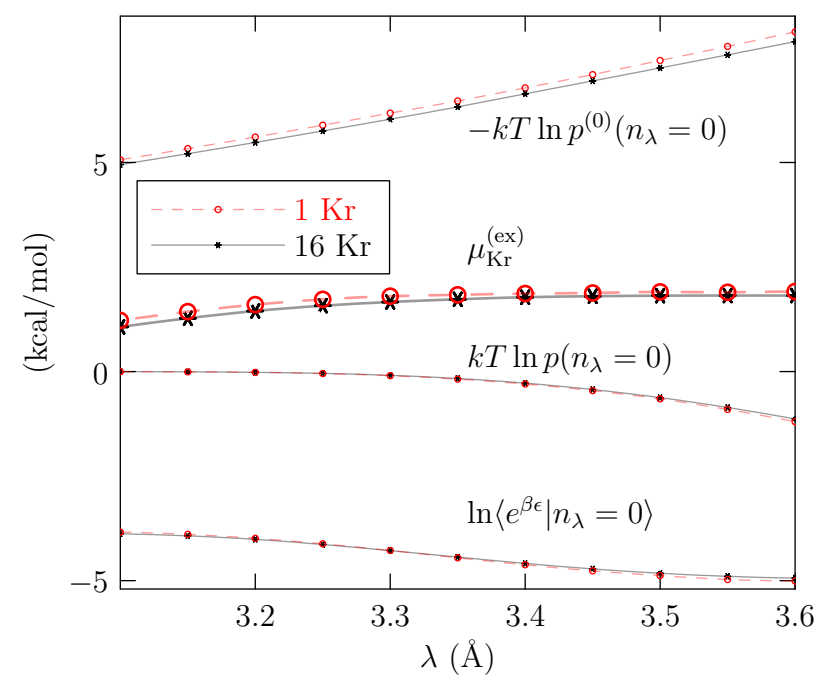

FIG. 4: Packing, chemical and outer-shell contributions to the hydration free energy of Kr were calculated numerically for a range of $\lambda$.

ural physical idea is to identify $\lambda$ with the van der Waals distance of closest approach. ${ }^{8 / 25}$ The individual contributions (FIG. 4) lead to free energies (FIG. 5) that are substantially independent of $\lambda$, provided $\lambda>3.4 \AA$. This displacement is less than the position of the principal maximum of $g_{\mathrm{KrO}}(r)$ and involves fewer than six (6) water molecules (FIG. 3).

Substituting these directly calculated numerical values for the various QCT contributions into Eq.1. we obtain a net $\mathrm{Kr}$ hydration free energy of $\mu_{\mathrm{Kr}}^{e x}=1.8-1.9 \mathrm{kcal} / \mathrm{mol}$,

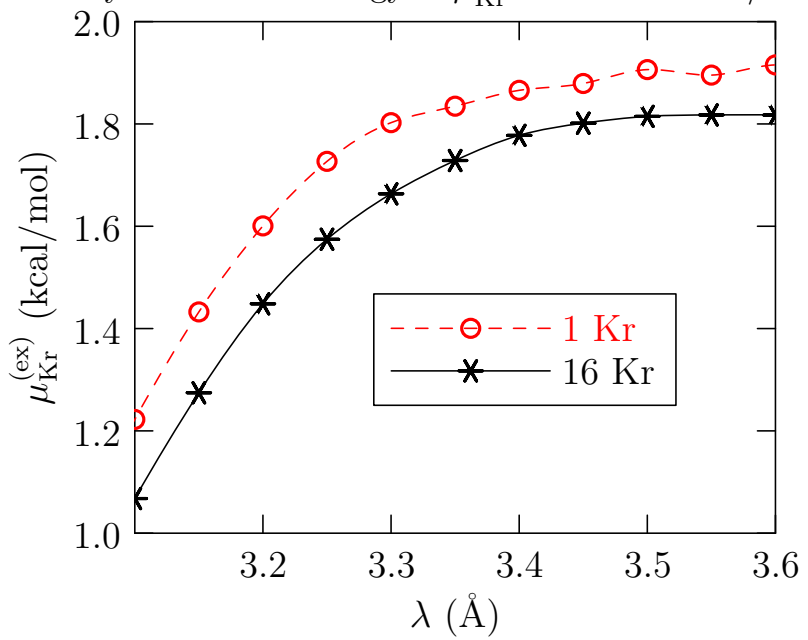

FIG. 5: Evaluations of hydration free energies on the basis of quasi-chemical theory for a range of inner-shell boundaries $(3.1 \AA<\lambda<3.6 \AA)$ for the two Kr concentrations. $\mu_{\mathrm{Kr}}^{(\mathrm{ex})}$ becomes insensitive to $\lambda$ for $3.4 \AA<\lambda<3.6 \AA$. The experimental value is $1.66 \mathrm{kcal} / \mathrm{mol}^{30}$

in reasonable agreement with the experimental value of $1.66 \mathrm{kcal} / \mathrm{mol}{ }^{30}$ Results for the two simulations, single $\mathrm{Kr}$ and multi-Kr, track each other. The multi-Kr results are distinctly lower (FIG. 5). The significant difference derives from the packing contribution.

The correlation function, $h_{\mathrm{KrKr}}(r)=g_{\mathrm{KrKr}}(r)-1$, observed in the multi-Kr simulation (FIG. 6) provides access to the osmotic second virial coefficient,

$$
B_{2}=-\frac{1}{2} \lim _{\rho_{\mathrm{Kr} \rightarrow 0}} \int h_{\mathrm{KrKr}}(r) \mathrm{d}^{3} r
$$

On the basis of the McMillan-Mayer theory, ${ }^{29}$ the integral can be identified thermodynamically as

$$
2 B_{2}=\lim _{\rho_{\mathrm{Kr} \rightarrow 0}}\left(\frac{\partial \beta \mu_{\mathrm{Kr}}^{(\mathrm{ex})}}{\partial \rho_{\mathrm{Kr}}}\right)_{T, \mu_{\mathrm{W}}}
$$

The partial derivative requires constancy of the water chemical potential, $\mu_{\mathrm{W}}$, but the calculations were done at constant pressure. The appropriate thermodynamic consideration of this distinction is 


$$
2 B_{2}=\lim _{\rho_{\mathrm{Kr} \rightarrow 0}}\left[\left(\frac{\partial \beta \mu_{\mathrm{Kr}}^{(\mathrm{ex})}}{\partial \rho_{\mathrm{Kr}}}\right)_{T, p}+\left(\frac{\partial \beta \mu_{\mathrm{Kr}}^{(\mathrm{ex})}}{\partial p}\right)_{T, \rho_{\mathrm{Kr}}}\left(\frac{\partial p}{\partial \rho_{\mathrm{Kr}}}\right)_{T, \mu_{\mathrm{W}}}\right]
$$

We note that

$$
\lim _{\rho_{\mathrm{Kr} \rightarrow 0}}\left(\frac{\partial \beta p}{\partial \rho_{\mathrm{Kr}}}\right)_{T, \mu_{\mathrm{W}}}=\lim _{\rho_{\mathrm{Kr} \rightarrow 0}}\left(\frac{\partial}{\partial \rho_{\mathrm{Kr}}}\left[\beta p_{\mathrm{W}}\left(T, \mu_{\mathrm{W}}\right)+\rho_{\mathrm{Kr}}+O\left(\rho_{\mathrm{Kr}}^{2}\right)\right]\right)_{T, \mu_{\mathrm{W}}}=1
$$

reflecting ideal osmotic pressure at low concentration. Referring again to Eq. (5), we use the notation

$$
v_{\mathrm{Kr}}^{(\mathrm{ex})}=\lim _{\rho_{\mathrm{Kr} \rightarrow 0}}\left(\frac{\partial \mu_{\mathrm{Kr}}^{(\mathrm{ex})}}{\partial p}\right)_{T, \rho_{\mathrm{Kr}}}
$$

for the excess contribution to the partial molar volume of the solute. Combining, we obtain

$$
2 B_{2}=\lim _{\rho_{\mathrm{Kr} \rightarrow 0}}\left(\frac{\partial \beta \mu_{\mathrm{Kr}}^{(\mathrm{ex})}}{\partial \rho_{\mathrm{Kr}}}\right)_{T, p}+v_{\mathrm{Kr}}^{(\mathrm{ex})}
$$

in the infinite dilution limit. For water, $\frac{31}{31}$ the ideal contribution to these partial molar volumes is about 1 $\mathrm{cm}^{3} / \mathrm{mol}$. For noble gases in water, the partial molar volumes are positive, and $v_{\mathrm{Kr}}^{(\mathrm{ex})}$ is about $33 \mathrm{~cm}^{3} / \mathrm{mol}-1$ $\mathrm{cm}^{3} / \mathrm{mol}=32 \mathrm{~cm}^{3} / \mathrm{mol}^{[32}$ The present simulation results yield a value of $v_{\mathrm{Kr}}^{(\mathrm{ex})} \approx 39 \mathrm{~cm}^{3} / \mathrm{mol}$, obtained by direct differencing of average volumes of simulation (at constant pressure) with/without a single Kr atom. Similarly, the leading term on the right-side of Eq. 8 is roughly estimated as $-160 \mathrm{~cm}^{3} / \mathrm{mol}$, using $\Delta \beta \mu_{\mathrm{Kr}} \approx-0.13$ (FIG. 5 ) and the observed $\Delta \rho_{\mathrm{Kr}} \approx 4.8 \times 10^{-4} \AA^{-3}$. The combined right-side of Eq. (8) is then about $-120 \mathrm{~cm}^{3} / \mathrm{mol}$.

Our direct analysis of the correlation function integral (FIG. 7) utilizes the extrapolation procedure advocated

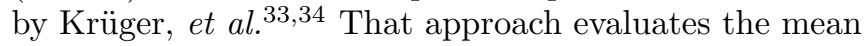
and variance of $\mathrm{Kr}$ occupancy $\left(n_{\mathrm{Kr}}\right)$ of a sphere of radius $R$, which is a sub-volume of the simulation cell. That sphere is the basis of a grand canonical ensemble with variable $n_{\mathrm{Kr}}$. Being tied to a grand canonical construction, the data analysis then corresponds directly to the McMillan-Mayer theory theoretical development, which proceeds through a grand ensemble. ${ }^{29}$ The formulae derived on that basis

$$
-2 B_{2}=\lim _{R \rightarrow \infty} 4 \pi \int_{0}^{2 R} h_{\mathrm{KrKr}}(r) w(r / 2 R) r^{2} \mathrm{~d} r,
$$

with

$$
w(x)=1-\left(\frac{3}{2}\right) x+\left(\frac{1}{2}\right) x^{3}
$$

are easy to use with conventionally available distribution functions. The $1 / 2 R=0$ extrapolated value (FIG. 7)

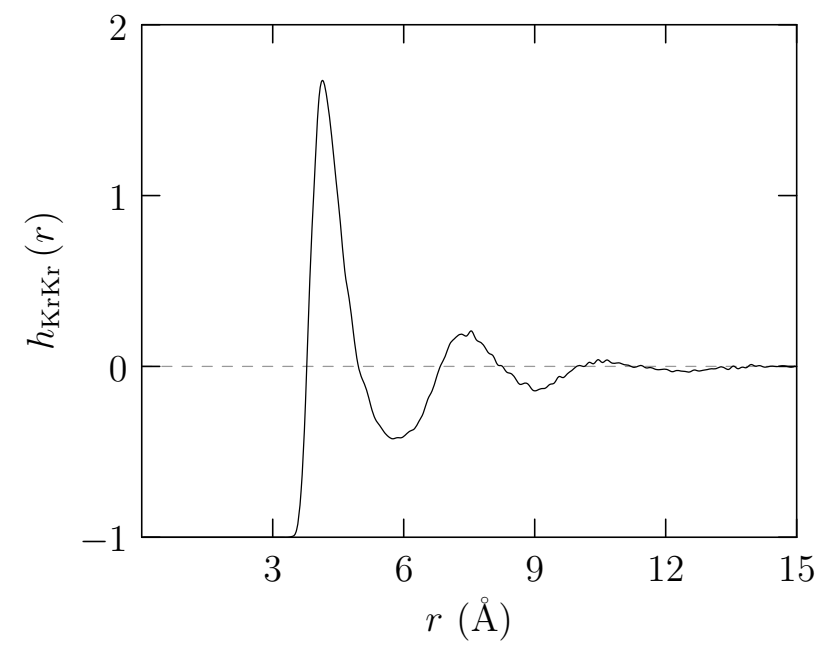

FIG. 6: For the multi-Kr simulation, the Kr-Kr correlation function, $h_{\mathrm{KrKr}}(r)=g_{\mathrm{KrKr}}(r)-1$, as in FIG. 1. but viewed more globally. Note the accessible $r$-range. The second- and third-shell structures are distinct, but quantitatively much less structured than the results of Watanabe and Andersen.

agrees precisely with Eq. (8) to the significant figures given there, the net value being $B_{2} \approx-60 \mathrm{~cm}^{3} / \mathrm{mol}$. In contrast, we found that direct integration of the distribution functions, or the $k \rightarrow 0$ extrapolation of the spatial Fourier transform, did not provide practical evaluations of $\mathrm{B}_{2}$.

\section{CONCLUSION}

Though $\mathrm{Rb}^{+}$is isoelectronic with $\mathrm{Kr}$, the solution structure is vastly different, and convincing inter-relation of these cases requires serious statistical mechanical theory! 1 Nevertheless, 20 the quasi-chemical approach evaluates the hydration free energy of $\operatorname{Kr}(\mathrm{aq})$ successfully, and in physical terms. This approach also obtains free energy differences reflecting $\mathrm{Kr}-\mathrm{Kr}$ interactions at higher concentrations (FIG. 5). Those differences are negative changes in hydration free energies with increasing concentrations at constant pressure. The changes are due to a slight reduction of unfavorable packing contributions 


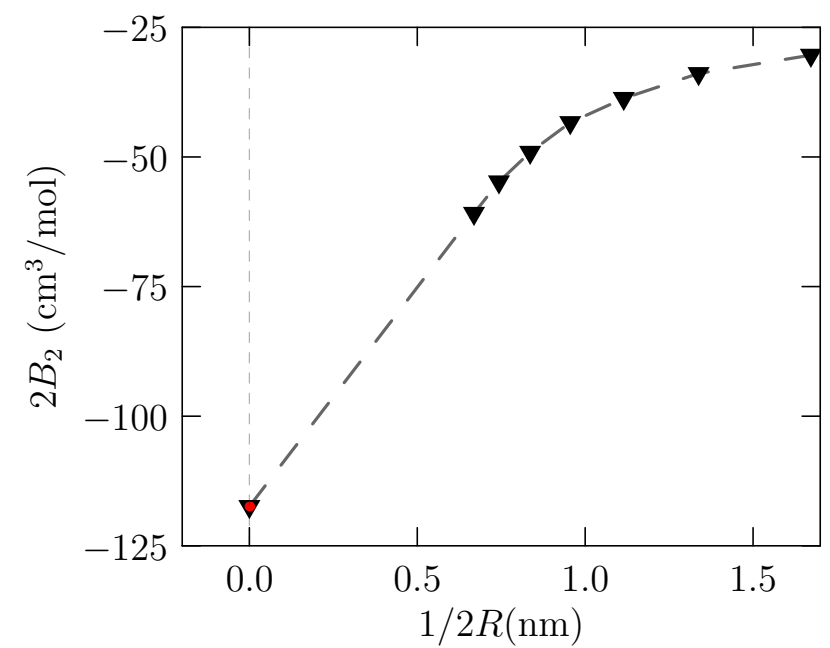

FIG. 7: Extrapolation (Eq. (9) following Krüger, et al! Computed values for $1 / 2 R>0$ were utilized to obtain a leastsquares-fit polynomial to quadratic order in $1 / 2 R$. The symbol at $1 / 2 R=0$ is the extrapolated value and it agrees precisely with Eq. (8) to the significant figures given there. in the higher concentration case. The observed $\mathrm{Kr}-\mathrm{Kr}$ distributions, analyzed with the extrapolation procedure of Krüger, et al! ${ }^{33 / 34}$ (FIG. 7), yield $B_{2} \approx-60 \mathrm{~cm}^{3} / \mathrm{mol}$. The thermodynamic analysis interconnecting these two approaches shows that they are closely consistent with each other. This provides support for both approaches.

\section{ACKNOWLEDGEMENT}

We thank J. D. Weeks for telling us of Ref. 33. Sandia is a multiprogram laboratory operated by Sandia Corporation, a Lockheed Martin Company, for the U.S. Department of Energy's National Nuclear Security Administration under Contract No. DE-AC04-94AL8500. The financial support of Sandia's LDRD program and the Gulf of Mexico Research Initiative (Consortium for Ocean Leadership Grant SA 12-05/GoMRI-002) is gratefully acknowledged.
* Electronic address: michaud@sandia.gov

† Electronic address: dubravko.sabo@nyu.edu

¥ Electronic address: lpratt@tulane.edu

$\S$ Electronic address: slrempe@sandia.gov

1 Sabo, D., Jiao, D., Varma, S., Pratt, L., and Rempe, S. (2013) Case study of $\mathrm{Rb}^{+}(\mathrm{aq})$, quasi-chemical theory of ion hydration, and the no split occupancies rule. Ann. Rep. Section C(Physical Chemistry) 109, 266-278.

2 Filipponi, A., Bowron, D., Lobban, C., and Finney, J. (1997) Structural Determination of the Hydrophobic Hydration Shell of Kr. Phys. Rev. Letts. 79, 1293-1296.

3 Bowron, D., Filipponi, A., Roberts, M., and Finney, J. (1998) Hydrophobic Hydration and the Formation of a Clathrate Hydrate. Phys. Rev. Letts. 81, 4164-4167.

${ }^{4}$ Watanabe, K., and Andersen, H. C. (1986) Molecular dynamics study of the hydrophobic interaction in an aqueous solution of krypton. J. Phys. Chem. 90, 795-802.

5 Kennan, R. P., and Pollack, G. L. (1990) Pressure dependence of the solubility of nitrogen, argon, krypton, and xenon in water. J. Chem. Phys. 93, 2724-2735.

6 Pfund, D. M., Darab, J. G., Fulton, J. L., and Ma, Y. (1994) An XAFS Study of Strontium Ions and Krypton in Supercritical Water. J. Phys. Chem. 98, 13102-13107.

7 Ashbaugh, H. S., Asthagiri, D., Pratt, L. R., and Rempe, S. B. (2003) Hydration of krypton and consideration of clathrate models of hydrophobic effects from the perspective of quasi-chemical theory. Biophys. Chem. 105, 323-338.

8 Asthagiri, D., Ashbaugh, H., Piryatinski, A., Paulaitis, M., and Pratt, L. (2007) Non-van der Waals treatment of the hydrophobic solubilities of $\mathrm{CF}_{4}$. J. Am. Chem. Soc. 129, 10133-10140.

9 Pratt, L. R., and Rempe, S. B. (1999) Quasi-chemical theory and implicit solvent models for simulations. AIP Con- ference Proceedings 492, 172-201.

10 Paulaitis, M. E., and Pratt, L. (2002) Hydration theory for molecular biophysics. Adv. Prot. Chem. 62, 283-310.

11 Beck, T. L., Paulaitis, M. E., and Pratt, L. R. The Potential Distribution Theorem and Models of Molecular Solutions; Cambridge University Press, 2006.

12 Chaudhari, M. I., Holleran, S. A., Ashbaugh, H. S., and Pratt, L. R. (2013) Molecular-scale hydrophobic interactions between hard-sphere reference solutes are attractive and endothermic. Proc. Natl. Acad. Sci. USA 110, 2055720562.

13 Koga, K. (2013) Osmotic second virial coefficient of methane in water. J. Phys. Chem. B 117, 12619-12624.

14 Van Der Spoel, D., Lindahl, E., Hess, B., Groenhof, G., Mark, A. E., and Berendsen, H. J. C. (2005) GROMACS: Fast, flexible, and free. J. Comp. Chem. 26, 1701-1718.

15 Hess, B., Bekker, H., and Berendsen, H. (1997) LINCS: a linear constraint solver for molecular simulations. J. Comp. Chem. 8, 1463-1472.

16 Nosé, S. (1984) A molecular dynamics method for simulations in the canonical ensemble. Mol. Phys. 52, 255-268.

17 Parrinello, M., and Rahman, A. (1981) Polymorphic transitions in single crystals: A new molecular dynamics method. J. Appl. Phys. 52, 7182-7190.

18 Berendsen, H., and Grigera, J. R. (1987) The missing term in effective pair potentials. J. Phys. Chem. 91, 6269-6271.

19 Jorgensen, W. L., and Maxwell, D. S. (1996) Development and testing of the OPLS all-atom force field on conformational energetics and properties of organic liquids. J. Am. Chem. Soc. 118, 11225-11236.

20 Asthagiri, D., Dixit, P., Merchant, S., Paulaitis, M., Pratt, L., Rempe, S., and Varma, S. (2010) Ion selectivity from local configurations of ligands in solutions and ion channels. Chem. Phys. Letts. 485, 1-7. 
21 Rogers, D. M., Jiao, D., Pratt, L., and Rempe, S. B. (2012) Structural models and molecular thermodynamics of hydration of ions and small molecules. Annu. Rep. Comput. Chem. 8, 71-128.

22 Paliwal, A., Asthagiri, D., Pratt, L. R., Ashbaugh, H. S., and Paulaitis, M. E. (2006) An analysis of molecular packing and chemical association in liquid water using quasichemical theory. J. Chem. Phys. 124, 224502.

23 Shah, J. K., Asthagiri, D., Pratt, L. R., and Paulaitis, M. E. (2007) Balancing local order and longranged interactions in the molecular theory of liquid water. J. Chem. Phys. 127, 144508.

${ }^{24}$ Chempath, S., and Pratt, L. R. (2009) Distribution of Binding Energies of a Water Molecule in the Water LiquidVapor Interface. J. Phys. Chem. B 113, 4147-4151.

25 Asthagiri, D., Merchant, S., and Pratt, L. R. (2008) Role of attractive methane-water interactions in the potential of mean force between methane molecules in water. J. Chem. Phys. 128, 244512.

${ }^{26}$ Sabo, D., Varma, S., Martin, M. G., and Rempe, S. B. (2008) Studies of the thermodynamic properties of hydrogen gas in bulk water. J. Phys. Chem. B 112, 867876.

27 Jiao, D., and Rempe, S. B. (2011) $\mathrm{CO}_{2}$ solvation free energy using quasi-chemical theory. J. Chem. Phys. 134, 224506 .
28 Chandler, D., Weeks, J. D., and Andersen, H. (1983) Van der Waals Picture of Liquids, Solids, and PhaseTransformations. Science 220, 787-794.

29 Zhang, W., You, X., and Pratt, L. R. (2013) Multiscale Theory in the Molecular Simulation of Electrolyte Solutions. J. Phys. Chem. B 118, 7730-7738.

30 Young, C. Hydrogen and deuterium (Solubility data series); Pergamon Press, 1981.

31 Pratt, L., and Pohorille, A. In Proceedings of the EBSA 1992 International Workshop on Water-Biomolecule Interactions; Palma, M. U., Palma-Vittorelli, M. B., and Parak, F., Eds.; Societá Italiana de Fisica, Bologna, 1993; pp 261-268.

32 Moore, J., Battino, T. R., Handa, Y. P., and Wilhelm, E. (1982) J. Chem. Eng. Data 27.

33 Krüger, P., Schnell, S. K., Bedeaux, D., Kjelstrup, S., Vlugt, T. J., and Simon, J.-M. (2012) Kirkwood-Buff Integrals for Finite Volumes. J. Phys. Chem. Letts. 4, 235-238.

34 Schnell, S. K., Englebienne, P., Simon, J.-M., Krüger, P., Balaji, S. P., Kjelstrup, S., Bedeaux, D., Bardow, A., and Vlugt, T. J. (2013) How to apply the Kirkwood-Buff theory to individual species in salt solutions. Chem. Phys. Letts. 582, 154-157. 